\section{Multi-facets of Serum Response Factor in the Cardiac Pathophysiology}

Received: July 07 2016; Accepted: July 08, 2016; Published: July 13, 2016

\section{Ankush Borlepawar ${ }^{1}$ and Ashraf Yusuf Rangrez ${ }^{1}$}

\author{
Internal Medicine III, Department of \\ Molecular Cardiology and Angiology, \\ University Medical Centre Kiel, Affiliated \\ to German Centre for Cardiovascular \\ Research (DZHK), Germany
}

Corresponding author: Dr. Ashraf Yusuf Rangrez organism through synchronous contraction and relaxation movements of the cardiac muscle. Homeostasis of cardiac muscle is thus necessary for normal heart functioning. Fine tuning of the cardiac muscle is maintained by numerous signal transduction pathways which are not only responsible for physiological functions, but pathological lesions as well. Many intrinsic or extrinsic stimuli like physical stress, pressure overload, hypertension, etc. disturbs cardiac homeostasis and affects signal transduction pathways causing disease phenotype such as hypertrophy, cardiomyopathy, and consequent heart failure. The most unique characteristic of cardiomyocytes is that they are terminally differentiated and do not grow in number postnatally, they only grow in size by lateral/longitudinal hypertrophy. Hypertrophy is also induced in cardiomyocytes to overcome acute wall tension caused by various biochemical stresses. At molecular level, several signalling pathways and interacting networks have been implicated with the heart's molecular response to physiological and/or pathological biomechanical stress, reviewed in detail elsewhere [1-4]. Calcineurin, a $\mathrm{Ca}^{2+}$-calmodulin activation dependent serine-threonine phosphatase is one of the key signaling molecules strongly linked to cardiac hypertrophy [5-8]. Its prohypertrophic effects are well established through series of in vitro and in vivo studies and is highly correlated with human patients of cardiac hypertrophy and cardiomyopathy $[6,9]$. Moreover, Calcineurin signaling is found to be interconnected with many other important hypertrophic pathways, such as those controlled by glycogen synthase kinase (GSK) $3 \beta$ and mitogenactivated protein (MAP) kinase signaling $[3,10]$. Interestingly, Rho family of small GTPase proteins, consisting of Rho, Rac, and $\mathrm{Cdc4} 2$ subfamilies regulate the sarcomere organization in cardiomyocytes, one of the hallmarks of hypertrophy [11]. These GTPase effectors are also upstream of a very important cardiac signaling molecule, serum response factor (SRF) $[12,13]$.

SRF is a highly conserved, ubiquitously expressed, and multifunctional founding member of the MADS (MCM1, Agamous, Deficiens, and SRF) box family of transcription factors, that has long been considered central to several regulatory complexes in muscle and non-muscle cells [14]. SRF regulates the expression of many muscle-specific and mitogen-responsive genes including some of the hypertrophy markers such as atrial/brain natriuretic peptides (ANF/BNP), Myosin heavy chains and $\alpha$-skeletal/cardiac actin, through binding to single or multiple consensus CArG box $(C C[A / T] 2 A[A / T] 3 G G)$ element in their promoter or enhancer sequences [15]. SRF in addition to affecting its target gene expression, also recruits different transcription factors in the heart, such as, cardiac NK2 Homeobox 5 (Nkx2.5), Transcriptional enhancer factor 1 (TEF-1), cardiac GATA family factors, and cofactors like myocardin, and of myocardin-related transcription factors [16-20]. SRF is therefore at the juncture of multiple signaling pathways that binds to serum response element in the promoter region of target genes and regulates the activity of many immediate-early genes, thereby participating in cell cycle regulation, apoptosis, cell growth, and cell differentiation in other cell types, and cell growth and homeostasis in cardiomyocytes.

Further insights into cardiac role of SRF were deduced from lossand gain-of-function approaches in vivo in mice. Classical global deletion of Srf leads to a very early embryonic lethality in mice, 
with a prominent defect in mesoderm formation, which hampered further evaluation of cardiac role of SRF [21]. To overcome this problem, Parlakian et al. has generated a mutant mouse line with targeted deletion of SRF in the heart [22]. They found that mice with early depleted cardiac SRF expression display severe cardiac defects, impaired expression of critical cardiac transcription factors such as Nkx2.5/GATA4/myocardin, and die between embryonic day 10.5 (E10.5) and E13.5 of development [22]. Similarly, Miano et al., generated mice where SRF was knocked out in $>80 \%$ of cardiomyocytes and $>50 \%$ of vascular smooth muscle cells using SM22alpha-Cre-mediated excision of promoter and first exon of SRF. They also noticed both cardiac and vascular defects such as highly disorganized sarcomere, defective actin/ intermediate filament bundles, and cardiac looping resulting embryonic death at E11.5. On the other hand, disruption of SRF in adult heart using a heart-specific Tamoxifen- inducible Cre recombinase led to progressive dilated cardiomyopathy and heart failure due to decreased expression of proteins involved in force generation and transmission, low levels of polymerized actin, and changes in cytoarchitecture without hypertrophic compensation [23]. Moreover, the symptoms observed in SRF-deficient mice at adolescence resemble morphological and clinical features of acquired dilated cardiomyopathy in humans. Furthermore, cardiac-sustained overexpression of SRF in transgenic mice is sufficient to develop cardiac hypertrophy and cardiomyopathy [24], whereas, overexpression of dominant-negative form of SRF in mice causes dilated cardiomyopathy [25]. These findings also have physiological and pathological relevance since the basal expression of SRF protein is found to be increased in old compared to young adult rat hearts [26], and an increased expression of an alternatively spliced dominant-negative isoform of SRF was observed in failing human hearts [27]. Taken together, findings from transgenic in vivo mouse models further strengthens the view that SRF is one of the downstream effectors of the signaling pathways involved in the induction of cardiac hypertrophy.

SRF has recently been implicated in the regulation and biogenesis of certain microRNAs [28]. miR-1, miR-21 and miR-133, all of which have established cardiac role, were found to be under direct regulation of SRF, adding not only another layer of complexity, but also provide an opportunity to explore for the therapeutic potential of SRF [28]. All these findings together undeniably demonstrate that SRF is crucial for cardiomyogenesis, adult cardiac function and integrity, and acts as a global regulator of multiple developmental genes and signaling cascades. SRF thus pose a strong therapeutic target for the treatment of cardiac diseases like cardiac hypertrophy, dilated cardiomyopathy and progressive heart failure. To revisit the existing knowledge, and the identification and characterization of yet unknown activators, inhibitors, and upstream and downstream effectors of this cardinal cardiac signaling molecule will therefore increase the possibilities of finding suitable drug molecule against the chronic cardiac diseases. 


\section{References}

1 Frey N, Olson EN (2003) Cardiac hypertrophy: The good, the bad and the ugly. Annu Rev Physiol 65: 45-79.

2 Frey N, Katus HA, Olson EN, Hill JA (2004) Hypertrophy of the heart: a new therapeutic target? Circulation 109: 1580-1589.

3 Vega RB, Bassel-Duby R, Olson EN (2003) Control of cardiac growth and function by calcineurin signaling. J Biol Chem 278: 36981-36984.

4 Olson EN (2004) A decade of discoveries in cardiac biology. Nat Med 10: $467-474$

5 Molkentin JD, Lu JR, Antos CL, Markham B, Richardson J, et al. (1998) A calcineurin-dependent transcriptional pathway for cardiac hypertrophy. Cell 93: 215-228.

6 Lim HW, De Windt LJ, Steinberg L, Taigen T, Witt SA, et al. (2000) Calcineurin expression, activation, and function in cardiac pressureoverload hypertrophy. Circulation 101: 2431-2437.

7 Sussman MA, Lim HW, Gude N, Taigen T, Olson EN, et al. (1998) Prevention of cardiac hypertrophy in mice by calcineurin inhibition. Science 281: 1690-1693.

8 Wilkins BJ, Dai YS, Bueno OF, Parsons SA, Xu J, et al. (2004) Calcineurin/ NFAT coupling participates in pathological, but not physiological, cardiac hypertrophy. Circ Res 94: 110-118.

9 Wilkins BJ, Molkentin JD (2002) Calcineurin and cardiac hypertrophy: where have we been? Where are we going? J Physiol 541: 1-8.

10 Vega RB, Yang J, Rothermel BA, Bassel-Duby R, Williams RS (2002) Multiple domains of MCIP1 contribute to inhibition of calcineurin activity. J Biol Chem 277: 30401-30407.

11 Hoshijima M, Sah VP, Wang YB, Chien KR, Brown JH (1998) The low molecular weight GTPase rho regulates myofibril formation and organization in neonatal rat ventricular myocytes - Involvement of Rho kinase. J Biol Chem 273: 7725-7730.

12 Seeger TS, Frank D, Rohr C, Will R, Just S, et al. (2010) Myozap, a novel intercalated disc protein, activates serum response factordependent signaling and is required to maintain cardiac function in vivo. Circ Res 106: 880-890.

13 Rangrez AY, Bernt A, Poyanmehr R, Harazin V, Boomgaarden I, et al. (2013) Dysbindin is a potent inducer of RhoA-SRF-mediated cardiomyocyte hypertrophy. J Cell Biol 203: 643-656.

14 Shore P, Sharrocks AD (1995) The MADS-box family of transcription factors. Eur J Biochem 229: 1-13.

15 Miano JM (2003) Serum response factor: toggling between disparate programs of gene expression. J Mol Cell Cardiol 35: 577-593.
16 Belaguli NS, Sepulveda JL, Nigam V, Charron F, Nemer M, et al. (2000) Cardiac tissue enriched factors serum response factor and GATA-4 are mutual coregulators. Mol Cell Biol 20: 7550-7558.

17 Chen CY, Schwartz RJ (1996) Recruitment of the tinman homolog Nkx-2.5 by serum response factor activates cardiac alpha-actin gene transcription. Mol Cell Biol 16: 6372-6384.

18 Gupta M, Kogut P, Davis FJ, Belaguli NS, Schwartz RJ, et al. (2001) Physical interaction between the MADS box of serum response factor and the TEA/ATTS DNA-binding domain of transcription enhancer factor-1. J Biol Chem 276: 10413-10422.

19 Hauschka SD (2001) Myocardin, A novel potentiator of SRF-mediated transcription in cardiac muscle. Mol Cell 8: 1-2.

20 Wang DZ, Li S, Hockemeyer D, Sutherland L, Wang Z, et al. (2002) Potentiation of serum response factor activity by a family of myocardin-related transcription factors. Proc Natl Acad Sci USA 99: 14855-14860.

21 Arsenian S, Weinhold B, Oelgeschlager M, Ruther U, Nordheim A (1998) Serum response factor is essential for mesoderm formation during mouse embryogenesis. Embo J 17: 6289-6299.

22 Parlakian A, Tuil D, Hamard G, Tavernier G, Hentzen D, et al. (2004) Targeted inactivation of serum response factor in the developing heart results in myocardial defects and embryonic lethality. Mol Cell Biol 24: 5281-5289.

23 Parlakian A, Charvet C, Escoubet B, Mericskay M, Molkentin JD, et al. (2005) Temporally controlled onset of dilated cardiomyopathy through disruption of the SRF gene in adult heart. Circulation 112: 2930-2939.

24 Zhang X, Azhar G, Chai J, Sheridan P, Nagano K, et al. (2001) Cardiomyopathy in transgenic mice with cardiac-specific overexpression of serum response factor. American journal of physiology. Heart and circulatory physiology 280: H1782-H1792.

25 Zhang X, Chai J, Azhar G, Sheridan P, Borras AM, et al. (2001) Early postnatal cardiac changes and premature death in transgenic mice overexpressing a mutant form of serum response factor. J Biol Chem 276: 40033-40040.

26 Muller JG, Thompson JT, Edmonson AM, Rackley MS, Kasahara H, et al. (2002) Differential regulation of the cardiac sodium calcium exchanger promoter in adult and neonatal cardiomyocytes by Nkx2.5 and serum response factor. J Mol Cell Cardiol 34: 807-821.

27 Davis FJ, Gupta M, Pogwizd SM, Bacha E, Jeevanandam V, et al. (2002) Increased expression of alternatively spliced dominantnegative isoform of SRF in human failing hearts. American journal of physiology. Heart and circulatory physiology 282: H1521- 1533.

28 Zhang X, Azhar G, Helms SA, Wei JY (2011) Regulation of cardiac microRNAs by serum response factor. J Biomed Sci 18: 15 . 